\section{MS13-05 Structure and dynamics of the polymyxin-resistance-associated response regulator PmrA in complex with the promoter DNA}

Chwan-Deng $\mathrm{Hsiao}^{1}$

1. Institute of Molecular Biology, Academia Sinica

email: hsiao@gate.sinica.edu.tw

PmrA, an OmpR/PhoB family response regulator (RR), manages genes for antibiotic resistance. Phosphorylation of OmpR/PhoB RR induces the formation of a symmetric dimer in the $\mathrm{N}$-terminal receive domain (REC), promoting $2 \mathrm{C}$-terminal DNA-binding domains (DBDs) to recognize the promoter DNA to elicit adaptive responses. Recently, determination of the KdpE-DNA complex structure revealed a unique asymmetric REC-DBD interface that may be necessary for transcription activation. Here, we report the $3.2-\AA$ resolution of the crystal structure of the PmrA-DNA complex, which reveals a similar yet different asymmetric REC-DBD interaction. However, this asymmetric interface is transiently populated in solution, as revealed by relaxation dispersion NMR, and is not crucial in supporting gene expression, as evidenced by the b-galactosidase reporter assay. The interdomain dynamics of the protein, together with the DBD-DBD interface, may help PmrA search for the most suitable conformation for interacting with RNA polymerase holoenzyme to activate downstream gene transcription.

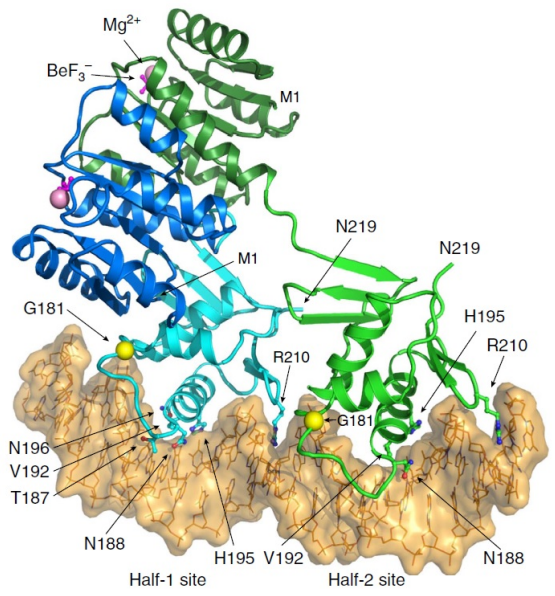

Figure 1. Structure of the $\mathrm{BeF}_{3}^{-}$-activated $\mathrm{PmrA}$ dimer in complex with the promoter DNA. The PmrA molecule that recognizes half-1 site is denoted as PmrA-1 and half-2 site as PmrA-2.

Keywords: Two-component systems, response regulator, crystal structure
MS14 Biomineralogical

crystallography and bioinspired

inorganic materials

Chairs: Wolfgang Schmahl, Fernandez-Diaz Lurdes

MS14-01 Learning from Sea Shells -

Bio-Inspired Approaches Toward

Mesoscale Architectures in Functional Spinel Oxides

Anna S. Schenk ${ }^{1}$, Sabine Eiben ${ }^{2}$, Miriam Goll ${ }^{1}$, Lukas Reith ${ }^{1}$, Elisabeth John ${ }^{1}$, Alex N. Kulak ${ }^{3}$, Fiona C. Meldrum ${ }^{3}$, Christina Wege $^{2}$, Sabine Ludwigs ${ }^{1}$

1. Institute of Polymer Chemistry, University of Stuttgart, Pfaffenwaldring 55, 70569 Stuttgart, Germany

2. Institute of Biomaterials and Biomolecular Systems, University of Stuttgart, Pfaffenwaldring 57, 70569 Stuttgart, Germany

3. School of Chemistry, University of Leeds, Woodhouse Lane, LS2 9JT, Leeds, United Kingdom

email: anna.schenk@ipoc.uni-stuttgart.de

Despite drawing on a limited pool of available elements, nature has developed sophisticated mechanisms to fabricate nanostructured minerals under environmental conditions. Biominerals characteristically represent organic/inorganic composite materials with hierarchical organization spanning multiple length scales, where these complex architectures often lead to astounding properties adapted to suit a specific function. Unsurprisingly, the study of biominerals has therefore inspired a substantial body of research activities, which are aiming to transfer key concepts of biological mineralization, e.g. the use of structure-directing organic matrices and confined reaction environments, into artificial systems. This approach is particularly exciting in view of the growing demand for eco-efficient synthetic routes for the preparation of technologically relevant materials showing inherent functional properties.

We here explore bio-inspired strategies to generate mesoscale architectures in spinel cobalt(II,III) oxide $\left(\mathrm{Co}_{3} \mathrm{O}_{4}\right)$ and related mixed metal spinels, where these compounds represent a highly promising class of materials for a wide range of applications, particularly in the field of heterogeneous catalysis. In this context, the catalytic activity of the functional oxide largely depends on its morphology, nanostructure and surface area. Specifically, we precipitate thermally unstable cobalt hydroxide carbonate precursors under biomimetic conditions in the presence of either synthetic water-soluble polymers with flexible chains or tobacco mosaic viruses (TMV), where the latter represent plant-derived nanoscopic biotemplates with a rigid-rod supramolecular structure. Calcination at relatively moderate temperatures converts the mineral precursors into the functional spinel oxide phase, while retaining the 
gross morphology induced by the structure-directing additives. We demonstrate that highly unusual $\mathrm{Co}_{3} \mathrm{O}_{4}$ structures such as thin films or micrometer-sized tubular superstructures composed of elongated nanoparticles can be generated based on bio-inspired concepts.

The products of the polymer- or virus-mediated mineralization reactions are analyzed before and after calcination using a range of imaging, spectroscopic and $\mathrm{x}$-ray scattering techniques. With the aim to ultimately establish structure-property relationships, the electrocatalytic activity of the resulting materials is investigated with respect to model reactions such as electrolytic water splitting.
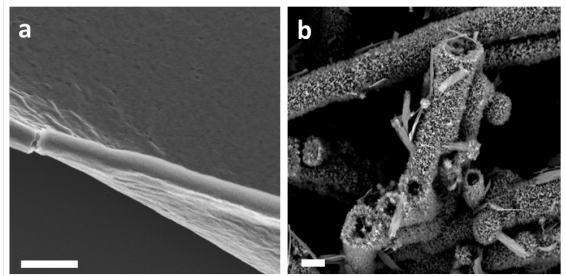

Figure 1. Bio-inspired mineralization of basic cobalt carbonate mediated by the water-soluble polyelectrolyte poly(vinylamine) (a) and tobacco mosaic viruses as biotemplates (b). Scale bars $=1$ $\mu \mathrm{m}$.

Keywords: bio-inspired mineralization, biotemplates, mesostructure, functional materials, small-angle $\mathrm{x}$-ray scattering

\section{MS14-02 Complex biomimetic}

self-assembly in simple inorganic precipitation systems

Matthias Kellermeier ${ }^{1}$

1. Material Physics, BASF SE, D-67056 Ludwigshafen, Germany

email: matthias.kellermeier@basf.com

Biomineralization provides spectacular examples for the concerted organization of inorganic matter into delicate superstructures with extraordinary properties designed for specific functions. Mimicking these processes in vitro is an overarching goal in material science and other areas of research. However, the currently achieved performance of bioinspired man-made materials does often not approach the natural counterpart yet - essentially due to the complexity of the phenomena leading to biomineral formation. Therefore, simplified model systems are needed to understand and ultimately control the fundamental mechanisms of self-assembly at various time and length scales.

Silica "biomorphs" are one such model system. They form upon crystallization of alkaline-earth metal carbonates in silica-rich environments at high $\mathrm{pH}$ and display structural and morphological features strongly reminiscent of biominerals and other living forms although no organic matter is involved in their formation. This contribution is meant to give an overview on the fascinating world of silica biomorphs and related structures. It is shown that the interplay of simplest components can lead to the spontaneous development of highly complex architectures if conditions are carefully controlled. A number of recent experimental observations shed light on the processes at work and suggest that dynamic reaction coupling drives self-assembly at the nanoscale, while even more peculiar mechanisms seem to govern the evolution of complex shapes at the micrometer level and beyond. Eventually, different approaches to benefit from the structural concept of silica biomorphs are highlighted along with possible implications for material science and early life detection.

\section{References}

[1] M. Kellermeier, H. Cölfen, J. M. Garcia-Ruiz, Eur. J. Mineral. 2012, 32, 5132.

[2] J. Opel, M. Hecht, K. Rurack, J. Eiblmeier, W. Kunz, H. Cölfen, M. Kellermeier, Nanoscale 2015, 7, 17434.

[3] J. Opel, F. P. Wimmer, M. Kellermeier, H. Cölfen, Nanoscale Horizons 2016, 1, 144. 